\section{Hydrodynamics and water waves}

An Introduction to Hydrodynamics and Water Waves. By Bernard Le Méhauté. Pp. viii +323 . (Springer: Berlin and New York, 1976.) DM 60.60; \$24.80.

AN obvious question to ask about this book is: what are the special contributions which it offers to an already well endowed literature on hydrodynamics and water waves? The declared aim of the book is to provide a text based on a set of lecture notes for engineering students, that introduces the mathematical aspects of fluid mechanics with explanations of physical meaning to help practising engineers to read mathematical texts and keep pace with new developments reported in scientific journals. It is intended, moreover, to have a wide range of application and in particular to appeal to "students of coastal, hydraulic and civil engineering, naval architecture, physical oceanography, marine geology and sedimentology".

Although it inevitably covers much well trodden ground, it fulfils its aims and has much to offer in clarity of explanation and in its orderly presentation of a broad selection of theoretical material. It may be disappointing, however, to those seeking a close contact with current engineering developments.

The text is divided into three main parts concerned, respectively, with the derivation of the basic equations of fluid motion, mathematical treatment of the equations, and water wave theories. There are two appendices, one dealing with wave spectra and associated statistical properties, and the other with problems of scale modelling.

Examples of clarity include well illustrated accounts of the distinctions between streamlines, stream tubes, streak lines and particle paths and of the significance of dilatation, shear and rotation in interpretating the kinematic equations. I specially commend the many well organised tabular summaries of key details and formulae which students will surely find very helpful and which offer convenient working references for practising engineers. Students should also appreciate the selection of illustrative problems at the end of each chapter for which answers are given in the back of the book. Although prominence is given to development of basic theory relevant to a broad treatment of water waves, material is included on flow in porous media and pipes, and on open channel hydraulics. Its claim to appeal to students in a wide range of professional ficlds therefore seems justified.
Although the book offers a clear and broadly based conceptual framework for the study of theoretical fluid dynamics the degree of contact with current engineering technology may be disappointing to those with a more practical turn of mind. A particular feature, for example, that I find rather frustrating is the almost total absence of cited references. There are lists called "References" at the end of each part but these might more appropriately be headed "Recommended background reading", since there are hardly any explicit references to these works in the text. The reader is therefore repeatedly tantalised by the introduction of complex ideas such as tensors, conformal mapping, ship waves and diffraction theory which are only very briefly discussed without mention of any references for further study.

N. Hogben

N. Hogben is a Senior Principal Scientific Officer at the National Maritime Institute, Middlesex, UK.

\section{Acetylcholine, the model transmitter}

Biology of Cholinergic Function. Edited by A. M. Goldberg and I. Hanin. Pp. xiv +716. (Raven: New York, September 1976.) \$42.

Books for biomedical scientists often promise more than they actually provide because the titles of convenience they bear have been conjured up by ingenious editors who wish to confer (on a disparate collection of symposium papers, for instance) a suggestion of cohesiveness and completeness where none exists. It is a pleasure to be able to declare at the very outset that Biology of Cholinergic Function escapes this criticism: it really does provide everything-perhaps more than-its title offers. I find it difficult to believe that anyone who buys the book will be disappointed with his purchase, even though his intellectual enrichment will be bought at the cost of economic impoverishment to the tune of $£ 26$ at this morning's rate of dollar exchange.

Neurotransmission processes attract a good deal of attention nowadays, and deservedly so, but in the midst of our animated discussions concerning such lively topics as monoamines and mental illness, the reawakening of interest in the possible transmitter roles of ATP, substance $P$ and histamine and the enthusiasm in many quarters in an increasing range of transmitter amino acids, it is easy to ignore acetylcholine, the earliest, the best authenticated and the most pervasive mediator of them all. Cerebral control systems rarely lack cholinergic synapses even though recent attention may seem to have been largely focused on their more immediately glamorous non-cholinergic components. Work on acetylcholine has, however, continued apace during the years when other substances have occupied the centre of the stage; and no worker in, or student of, the field of chemical transmission can afford to ignore it. It is conveniently assembled for him within the substantial covers of this book.

This massive compilation (more than 700 pages) is encyclopaedic in scope with exhaustive and up-to-date surveys of the literature (papers published as recently as 1975 are referenced) and of recent experimental findings. The main body of the text-18 chapters in all-discusses acetylcholine metabolism and actions in vertebrates and invertebrates, at subcellular and at whole organ levels, at peripheral and central sites, in muscle and in nerve. Those who champion the claims of noncholinergic transmitters would do well to pay particular attention to the chapter that discusses the interaction of cholinergic and non-cholinergic transmitter processes and to the group of four chapters that together form a section on cholinergically linked diseases. The book is enriched for the laboratory worker by the inclusion of two appendicular tables. One of them, nineteen pages long, provides information concerning the acetylcholine content and cholinesterase activity of the tissues. It lists the results of all the studies that have worked their way through into the literature. The other table, only two pages shorter, does the same for the actions of drugs on the acetylcholine system. These tables are described in the preface as compendia of 'cholinergic parameters' but, this semantic monstrosity apart, the literary style of the book as a whole is tolerable if not exactly memorable.

Multiauthor texts are prone to exhibit irregularities in style and in depth of treatment and to suffer from omissions and repetitions that irritate the busy reader. This volume cannot be faulted on these grounds because the twenty-nine contributors worked together as a team under the guidance of editors who clearly took their duties seriously. All twenty-nine authors live in the United States but this does not unfairly bias the book towards American workers and attitudes. I hope that this judgment is not unduly influenced by the fact that my own modest contributions to the subject of the book are not ignored.

James Crossland

James Crossland is Professor of Pharmacology at the University of Nottingham, $U K$. 